\title{
Long noncoding RNA ATB promotes the epithelial-mesenchymal transition by upregulating the miR-200c/Twist 1 axe and predicts poor prognosis in breast cancer
}

\author{
Rong-Hui Li ${ }^{1}$, Min Chen ${ }^{1,2}$, Jing Liu' ${ }^{1}$, Chang-Chun Shao ${ }^{1}$, Cui-Ping Guo ${ }^{3}$, Xiao-Long Wei ${ }^{4}$, Yao-Chen Li ${ }^{1}$, \\ Wen-He Huang ${ }^{3}$ and Guo-Jun Zhang $\mathbb{1}^{1,2}$
}

\begin{abstract}
Recent studies indicate that the long noncoding RNA ATB (IncATB) can induce the epithelial-mesenchymal transition (EMT) in cancer cells, but the specific cellular targets of IncATB require further investigation. In the present study, the upregulation of InCATB in breast cancer cells was validated in a TGF- $\beta$-induced EMT model. Gain- and loss-of-function studies demonstrated that InCATB enhanced cell migration, invasion and clonogenicity in vitro and in vivo. LncATB promoted the EMT by acting as a sponge for the miR-200 family and restoring Twist1 expression. Subsequently, the clinical significance of IncATB was investigated in a cohort of breast cancer patients $(N=131)$. Higher IncATB expression was correlated with increased nodal metastasis $(P=0.036)$ and advanced clinical stage $(P=0.011)$ as well as shorter disease-free survival $(P=0.043)$ and overall survival $(P=0.046)$. These findings define Twist1 as a major target of IncATB in the induction of the EMT and highlight InCATB as a biomarker in breast cancer patients.
\end{abstract}

\section{Introduction}

Over the past decade, great improvements have been made in the management of breast cancer, but patient outcomes still merit consideration based on the increasing rate of tumour incidence and the high rate of tumourspecific death due to recurrence and metastasis ${ }^{1-3}$. However, targeted therapy has proven to be an effective strategy for breast cancer treatment ${ }^{4,5}$. Therefore, identifying new targets that contribute to breast cancer malignancy, as well as elucidating the underlying molecular mechanisms, is urgently required.

At least $75 \%$ of the genome is transcribed into noncoding RNA, which has no protein-coding capacity ${ }^{6}$.

\footnotetext{
Correspondence: G-J. Zhang (gjzhang@xah.xmu.edu.cn)

'ChangJiang Scholar's Laboratory, Shantou University Medical College (SUMC), 515041 Shantou, Guangdong, China

${ }^{2}$ The Cancer Center, Xiang'an Hospital of Xiamen University, 2000 East Xiang'an Rd., Xiang'an, Xiamen, China

Full list of author information is available at the end of the article.

Edited by B. Rotblat
}

Generally, long noncoding RNAs (lncRNAs) are longer than 200 nucleotides $^{7-9}$ and undergo transcription, 5'capping, polyadenylation and splicing under the control of RNA polymerase $\mathrm{II}^{6}$. LncRNAs participate in the regulation of gene expression at the transcriptional and posttranscriptional levels ${ }^{10-12}$. LncRNAs drive various cancerous phenotypes, including characteristics associated with immortality, cancer cell proliferation, anti-apoptosis and invasion, through the interaction of these RNAs with other cellular macromolecules, such as DNA, protein, and RNA $^{13-15}$.

To our knowledge, several lncRNAs associated with breast cancer prognosis have been identified, including HOTAIR, which regulates cancer cell proliferation and invasion $^{16,17}$, MALAT1, which has been correlated with breast cancer metastasis ${ }^{18,19}, \mathrm{H} 19$, which is involved in various functions during the complex process of tumour progression $^{20-22}$, and other CCAT2 ${ }^{23,24}$, MCM3AP-AS1 and $\mathrm{PCAT}^{25}$. Very recently, using Gene Expression

\section{(c) The Author(s) 2018}

(c) (i) Open Access This article is licensed under a Creative Commons Attribution 4.0 International License, which permits use, sharing, adaptation, distribution and reproduction in any medium or format, as long as you give appropriate credit to the original author(s) and the source, provide a link to the Creative Commons license, and indicate if changes were made. The images or other third party material in this article are included in the article's Creative Commons license, unless indicated otherwise in a credit line to the material. If material is not included in the article's Creative Commons license and your intended use is not permitted by statutory regulation or exceeds the permitted use, you will need to obtain permission directly from the copyright holder. To view a copy of this license, visit http://creativecommons.org/licenses/by/4.0/. 
Omnibus (GEO) and the Cancer Genome Atlas (TCGA) database, Xu et al. ${ }^{25}$ group identified TINCR, LINC00511, and PPP1R26-AS1 as subtype-specific lncRNAs associated with HER-2, triple-negative and luminal B subtypes, respectively. Intriguingly, an increasing number of lncRNAs have been validated as potential breast cancer biomarkers, including the four potentially diagnostic lncRNAs RP11-434D9.1, LINC00052, BC016831, and $\mathrm{IGKV}^{26}$, and BCAR4 as a prognostic marker for tamoxifen resistance and tumour invasion ${ }^{24,27,28}$. Thus, all these findings highlight lncRNAs as potential biomarkers for breast cancer as well as the mechanisms contributing to breast cancer malignancy ${ }^{22,29-31}$.

LncATB, a noncoding RNA located on chromosome 14 (ENST00000493038) with a length of 2446-bp, was first reported as a noncoding transcript enriched in human hepatocellular carcinoma (HCC) cells after a TGF- $\beta$ stimulus in 2014, and also as a predictor for HCC patient survival $^{32}$. Since its discovery in HCC, lncATB has been linked as a novel potential diagnostic and prognostic biomarker to other human tumours, such as non-small cell lung cancer ${ }^{33}$, osteosarcoma ${ }^{34}$, colon cancer ${ }^{35}$, renal cell carcinoma ${ }^{36,37}$, gastric cancer $^{38,39}$, thyroid cancer ${ }^{40}$, oesophageal squamous cell carcinoma ${ }^{41}$ and glioma malignancy ${ }^{42}$. In an effort to explore the role of lncATB, we found when searching the NONCODE database (http://www.noncode.org) that the expression of lncATB was higher in the breast and ovary. Additional evidence has accumulated revealing the premetastatic role of lncATB in human cancer via acting as a sponge for the miR-200 family to thereby restore ZEB1and ZEB2 expression $^{32,34,43,44}$, restore ZNF217 expression $^{43}$ or promote IL-11 signalling ${ }^{32}$. Regarding the role of IncATB in breast cancer, a recent study reported that lncATB promoted trastuzumab resistance and invasiveness in Her2positive breast cancer cells by competitively binding miR$200 \mathrm{c}$ to restore the expression of the miR-200c target genes ZEB1 and ZNF-217, resulting in EMT-mediated drug resistance ${ }^{45}$. However, the roles and clinical significance of lncATB, the functions of lncATB in malignancy and the related molecular mechanisms in breast remain unclear.

In the present study, we aimed to explore the role of lncATB in the EMT and the relevant mechanisms of lncATB in breast cancer progression and metastases, and to further identify the clinical significance of lncATB in a breast cancer cohort.

\section{Results}

TGF- $\beta$ induced IncATB and altered EMT markers in breast cancer cells in vitro

To detect lncATB levels, qRT-PCR assays were used to determine the relative expression of lncATB in the normal human breast cell line MCF-10A and eight breast cancer cell lines. Compared to the normal breast cell line MCF-10A, the expression level of lncATB was higher in breast cancer cell lines. Even though the variation for the lncATB expression level was noticed in different breast cancer cell lines, however, the average expression level of lncATB was relative higher in four TNBC cell lines (MDA-MB-231, MDA-MB-436, BT-549 and BT-20) and two Her-2-positive cell lines (SKBR3 and MDA-MB-435) than two luminal A cell lines (MCF-7 and T47D) (Fig. 1a).

To determine whether lncATB can be regulated by TGF- $\beta$, we continuously treated MCF-7 cells with TGF- $\beta$ at various concentrations $(0,2.5,5,10$, or $20 \mathrm{ng} / \mathrm{ml})$ for $72 \mathrm{~h}$. The expression of lncATB was sufficiently upregulated with TGF- $\beta$ treatment in a dose-dependent manner (Fig. 1b) and treatment with $10 \mathrm{ng} / \mathrm{ml}$ TGF- $\beta$ resulted in a saturation level. And also the downregulation of $\mathrm{E}$ Cadherin (E-Cad) protein or upregulation of $\mathrm{N}$-cadherin $(\mathrm{N}-\mathrm{Cad})$ and Vimentin proteins was shown to be dosedependent regarding TGF- $\beta$ treatment (Fig. 1c). In addition, the $10 \mathrm{ng} / \mathrm{ml}$ TGF- $\beta$ treatment decreased the expression levels of an epithelial marker E-Cad, but increased the expression of the mesenchymal markers $\mathrm{N}$ Cad and Vimentin and the transcription factors ZEB1 and Twist1, and importantly, through knockdown lncATB rescued the TGF- $\beta$-induced dysregulation of those genes (Fig. 1d, e). Moreover, immunofluorescence staining revealed that $10 \mathrm{ng} / \mathrm{ml}$ TGF- $\beta$ reduced E-Cad expression and induced Vimentin expression in MCF-7 cells (Fig. 1e). These results demonstrated that TGF- $\beta$, an EMT inducer, induced lncATB and altered EMT markers in breast cancer cells in vitro.

\section{LncATB overexpression and knockdown promoted and suppressed EMT, respectively, in breast cancer cells}

To investigate the potential role of lncATB in the EMT process in breast cancer cells, we overexpressed (Supplementary Fig. S1a) or knocked down lncATB in breast cancer cell lines. Successful ectopic lncATB expression (84.4-fold increase in MCF-7-ATB stable cells, Fig. 2a) altered the expression of both epithelial and mesenchymal markers at both the protein (Fig. 2b, c) and transcriptional levels (Fig. 2d), e.g., upregulated ZEB1, Twist1, N-Cad and Vimentin and downregulated E-Cad were observed. Successful lncATB knockdown (88.7 and 82.9\% knockdown for lncATB using shATB-\#1 and shATB-\#2, respectively, in BT-549 cells, Fig. 2e) resulted in downregulated ZEB1, Twist 1 and Vimentin and upregulated E-Cad at both the protein (Fig. 2f, g) and transcriptional levels (Fig. 2h). Furthermore, as expected, immunofluorescence staining showed that E-Cad was decreased and Vimentin was increased in the MCF-7-ATB stable cells, whereas E-Cad was increased and Vimentin was decreased in the BT-549shATB stable cells (Fig. 2i). Additionally, other EMTrelated markers, such as MMP-2, MMP-9, Snail, and Slug, 
D

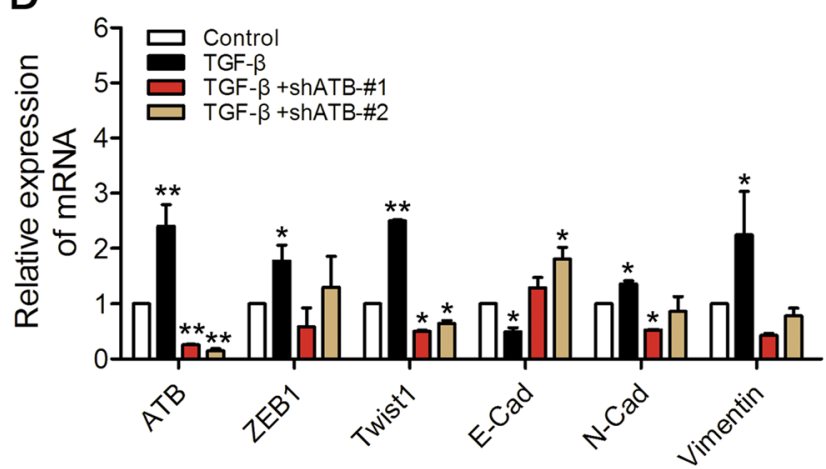

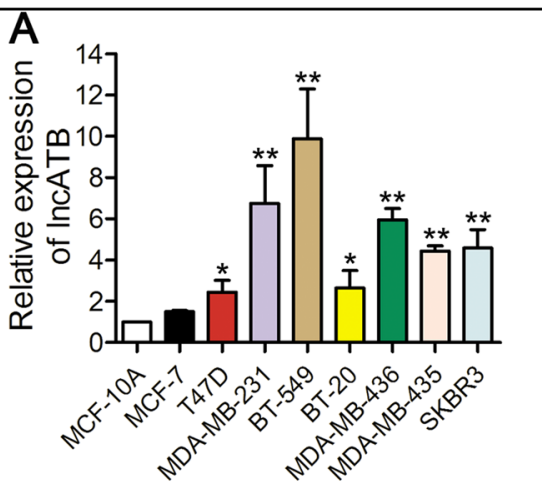

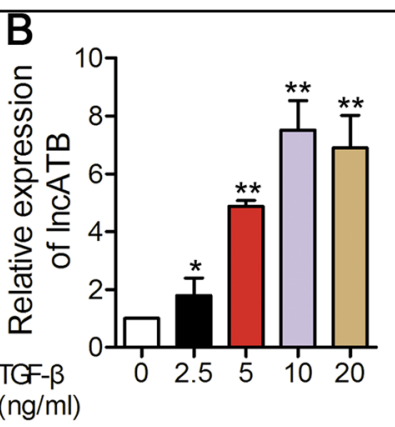

E

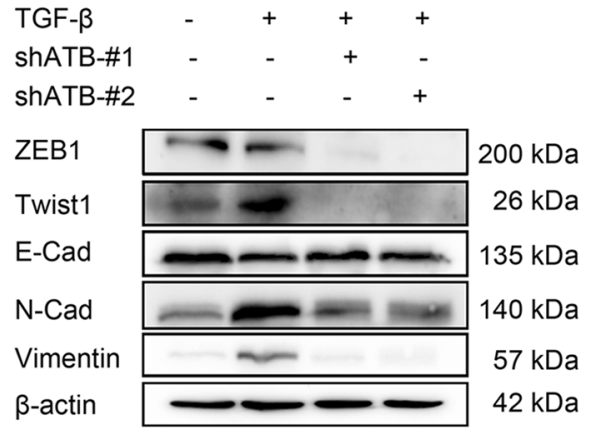

F

E-Cad

DAPI

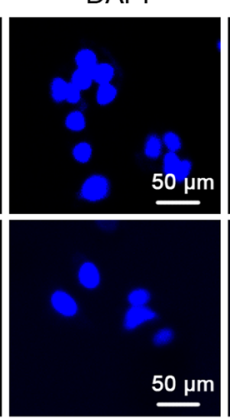

Merged

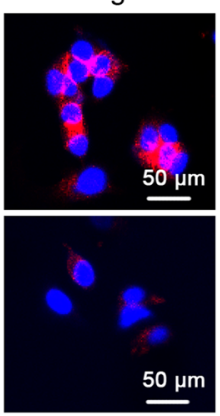

Vimentin

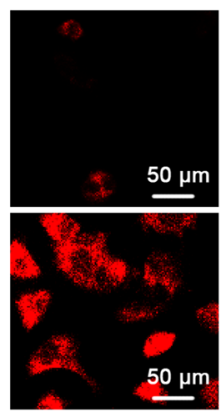

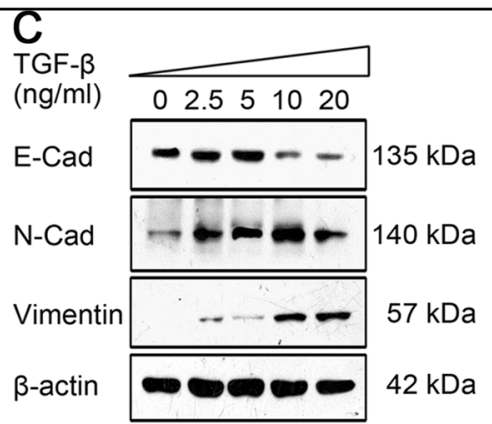

TGF- $\beta$

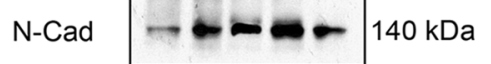

$\beta$-actin

$42 \mathrm{kDa}$

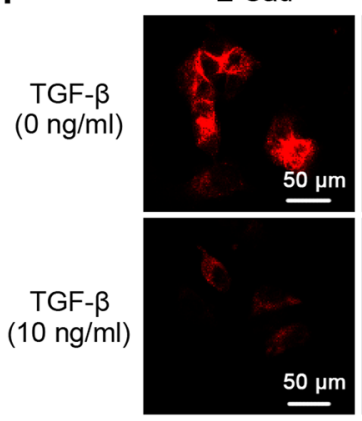

Fig. 1 TGF- $\beta$ induced IncATB and altered EMT markers in MCF-7 cells. a LncATB expression in different breast cancer cell lines. $\mathbf{b}$ Relative expression of InCATB in MCF-7 cells treated with TGF- $\beta$ at the indicated concentrations. c The protein levels of EMT markers determined by western blot analysis in MCF-7 cells treated with TGF- $\beta$ at the indicated concentrations. $\mathbf{d}$ Relative mRNA levels of IncATB and EMT markers measured by qRTPCR assays in MCF-7 cells treated with TGF- $\beta$ or both treated with TGF- $\beta$ and shATB-\#1/2. e Relative protein levels of EMT markers measured by western blot analysis in MCF-7 cells treated with TGF- $\beta$ or both treated with TGF- $\beta$ and shATB-\#1/2. $\mathbf{f}$ Immunofluorescence staining of E-Cad and Vimentin in MCF-7 cells treated with $10 \mathrm{ng} / \mu \mathrm{l} \mathrm{TGF-} \beta$. ${ }^{*} P<0.05$ and ${ }^{* *} P<0.01$

were assessed but did not have a significant relationship with IncATB (Supplementary Fig. S1b, c). These data indicated that the transcription factors ZEB1 and Twist1 were upregulated by lncATB and promoted the EMT in breast cancer cells.

\section{LncATB acts as a miR-200 family sponge}

We used the NCBI and miRBase programs to predict the miRNAs that potentially bind to lncATB. The analysis showed that lncATB has three potential binding sites for the miR-200 family. To further prove the direct binding between IncATB and the miR-200 family, we applied biotin-labelled lncATB RNA probes to pull down endogenous miR-200 family members in MCF-7 cells (Fig. 3a). The RNA pull-down experiment showed that the strongest association between members of the miR-200 family and lncATB was with miR-200c (13.8-fold), followed by miR-200b and miR-429 (Fig. 3b). Surprisingly, the expression levels of the miR-200 family members did not significantly change upon lncATB overexpression or 

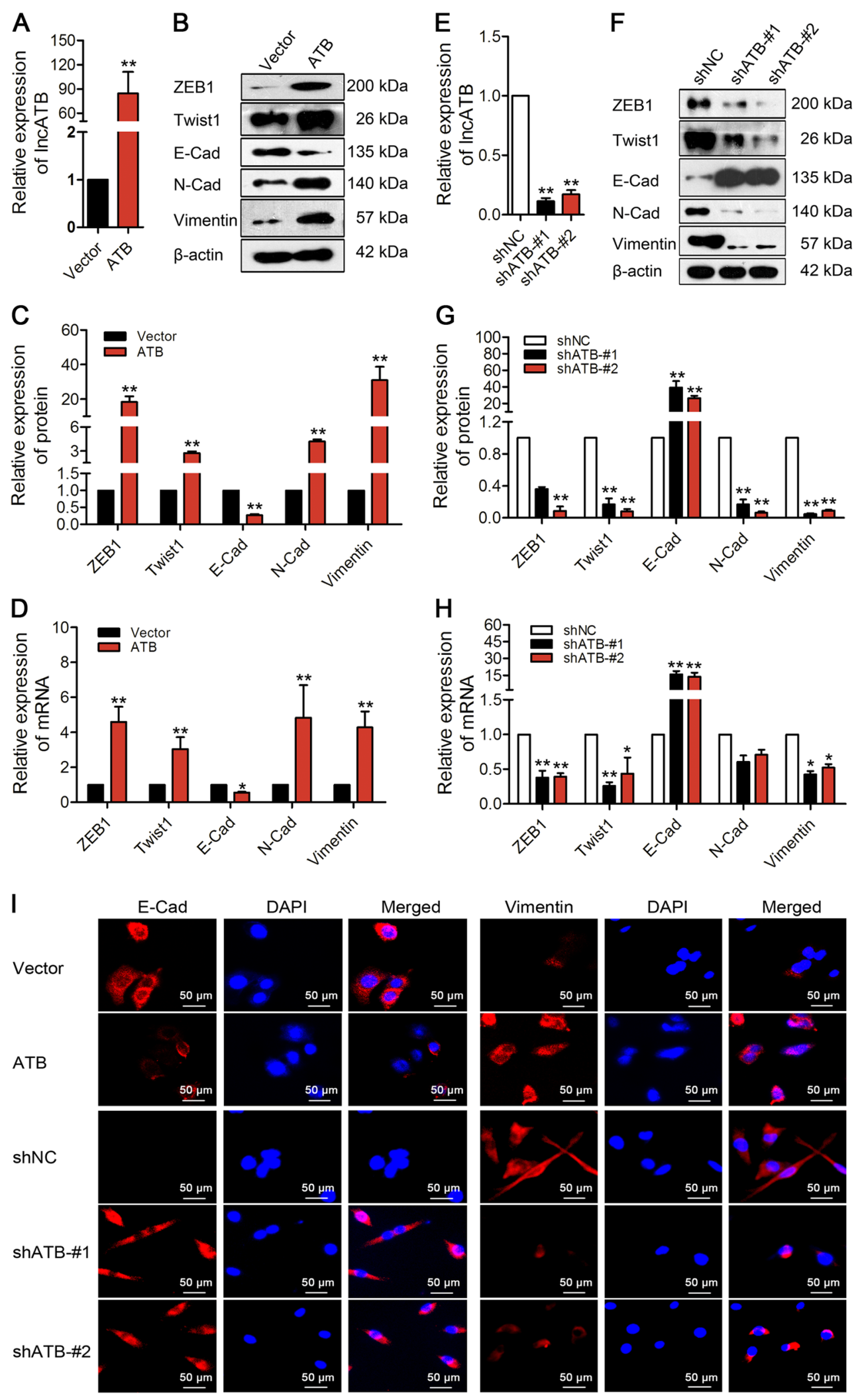

Fig. 2 (See legend on next page.) 
(see figure on previous page)

Fig. 2 LncATB overexpression increased mesenchymal makers and IncATB knockdown increased epithelial markers in breast cancer cells. a LncATB overexpression in stable MCF-7 cell clones. b, c The protein levels of EMT markers determined by western blot analysis in IncATBoverexpressing MCF-7 cells. d The mRNA levels of EMT markers in IncATB-overexpressing MCF-7 cells. e Knocking down IncATB in stable BT-549 cell clones. $\mathbf{f}, \mathbf{g}$ The protein levels of EMT markers determined by western blot analysis in IncATB-knockdown BT-549 cells. $\mathbf{h}$ The mRNA levels of EMT markers in IncATB-knockdown BT-549 cells. i Immunofluorescence staining of E-Cad and Vimentin in MCF-7-ATB and BT-549-shATB cells. ${ }^{*} P<0.05$ and ${ }^{* *} P<0.01$

deletion, indicating that lncATB may only be a sponge for miR-200 family (Supplementary Fig. S1d, e). Additionally, a luciferase reporter system, tdT/miR-200 family, was also used to confirm the competitive binding between lncATB and the miR-200 family. Firstly, we verified the activity of the reporter genes, which exhibited signalling in a dosedependent manner (Supplementary Fig. S2a-j). The results showed that the luciferase activity of $t d \mathrm{~T} / \mathrm{miR}$ 200b, tdT/miR-200c and tdT/miR-429 increased after lncATB overexpression in MCF-7 cells and decreased after lncATB deletion in BT-549 cells in a dose-dependent manner, while there was no significant difference in the luciferase activity of tdT/miR-200a or tdT/miR-141 (Supplementary Fig. S2k-t). The ectopic expression of miR-200b, miR-200c or miR-429 abrogated this increase (Fig. 3c-g), and conversely, the inhibition of miR-200b, miR-200c or miR-429 overcame this decrease (Fig. $3 \mathrm{~h}-\mathrm{l}$ ). All of these results demonstrated that lncATB acts as a miR200-family sponge in breast cancer cells, especially for miR-200c.

\section{LncATB restores the expression of Twist1 by binding to miR-200c}

When searching the microRNA databases miRBase, TargetScanHuman and the miRcode program, we confirmed that there is one binding site for miR-200c in the Twist1 3'UTR (Supplementary Table S1). Thus, we constructed two luciferase reporter vectors containing the wild-type or mutant Twist1 3'UTR. When miR-200c mimics was administered, the luciferase activity of WT reporter was decreased in a dose-dependent manner (Fig. 4a). In contrast to miR-200c mimics, the luciferase activity of WT reporter was conversely increased by adding miR-200c inhibitor (Fig. 4b). However, the luciferase activity of mutant reporter did not change to the miR-200c mimics or inhibitors. The results showed that lncATB-induced Twist1 mRNA (Fig. 4c) or protein and lncATB-suppressed E-Cad protein (Fig. 4e) expression in MCF-7 cells was abrogated by the ectopic expression of miR-200c. Conversely, in BT-549 cells with knockeddown lncATB, the decreased Twist1 mRNA (Fig. 4d) or protein and Vimentin protein (Fig. 4f) expression was rescued by inhibiting miR-200c. All of these data revealed the important role of lncATB in restoring Twist1 expression by acting as a sponge for miR-200c.

\section{LncATB promotes breast cancer cell proliferation, migration and invasion}

To directly test whether lncATB promotes breast cancer progression, we conducted proliferation, colony formation, wound-healing, and migration and invasion assays in the MCF-7-ATB and BT-549-shATB cells. We observed that the forced overexpression of lncATB expedited proliferation by 3 -fold (Fig. 5a) and increased the number of colonies by 4.6-fold (Fig. 5b). Furthermore, tumour cell migration and invasion were increased approximately twofold in the MCF-7-ATB cells, while the increase was abrogated when the cells were treated with miR-200c mimics (Fig. 5c, d).

The depletion of lncATB suppressed cell growth by approximately 2-fold (Fig. 6a) and decreased the number of colonies by 4-5-fold (Fig. 6b). Additionally, the depletion of lncATB reduced the migration and invasion ability by 2.5 -fold in BT-549 cells, while this decrease was rescued when the cells were treated with miR-200c inhibitor (Fig. 6c, d). All of these results implied that lncATB has the potential to promote breast cancer progression and metastasis.

\section{LncATB induces tumourigenesis in breast cancer tumour xenograft models in vivo}

To ascertain the effects of lncATB expression in tumourigenesis, we performed tumour xenograft studies with highly tumourigenic MDA-MB-231 cells in nude mice. Firstly, we detected the relative expression levels of lncATB and EMT markers. The results led to a conclusion similar to that above; i.e., when lncATB was depleted, the mRNA expression levels of ZEB1, Twist1 and Vimentin were increased while that of E-Cad was decreased (Supplementary Fig. S3a, b). Next, control or stably lncATB-depleted MDA-MB-231 cells were subcutaneously implanted into the left or right fourth breast of nude mice (Fig. 6e). Tumour growth was determined using callipers, and the tumour volume was calculated as $V=0.5 \times$ width $^{2} \times$ length. At 27 days after implantation, an 82 and $73 \%$ reduction in the tumour volume was observed in the lncATB-downregulated shATB-\#1 and shATB-\#2 groups, respectively, compared with the control (Fig. 6f). At the end of the observation period, the subcutaneous tumours were dissected and weighed. Our results showed that lncATB downregulation reduced 


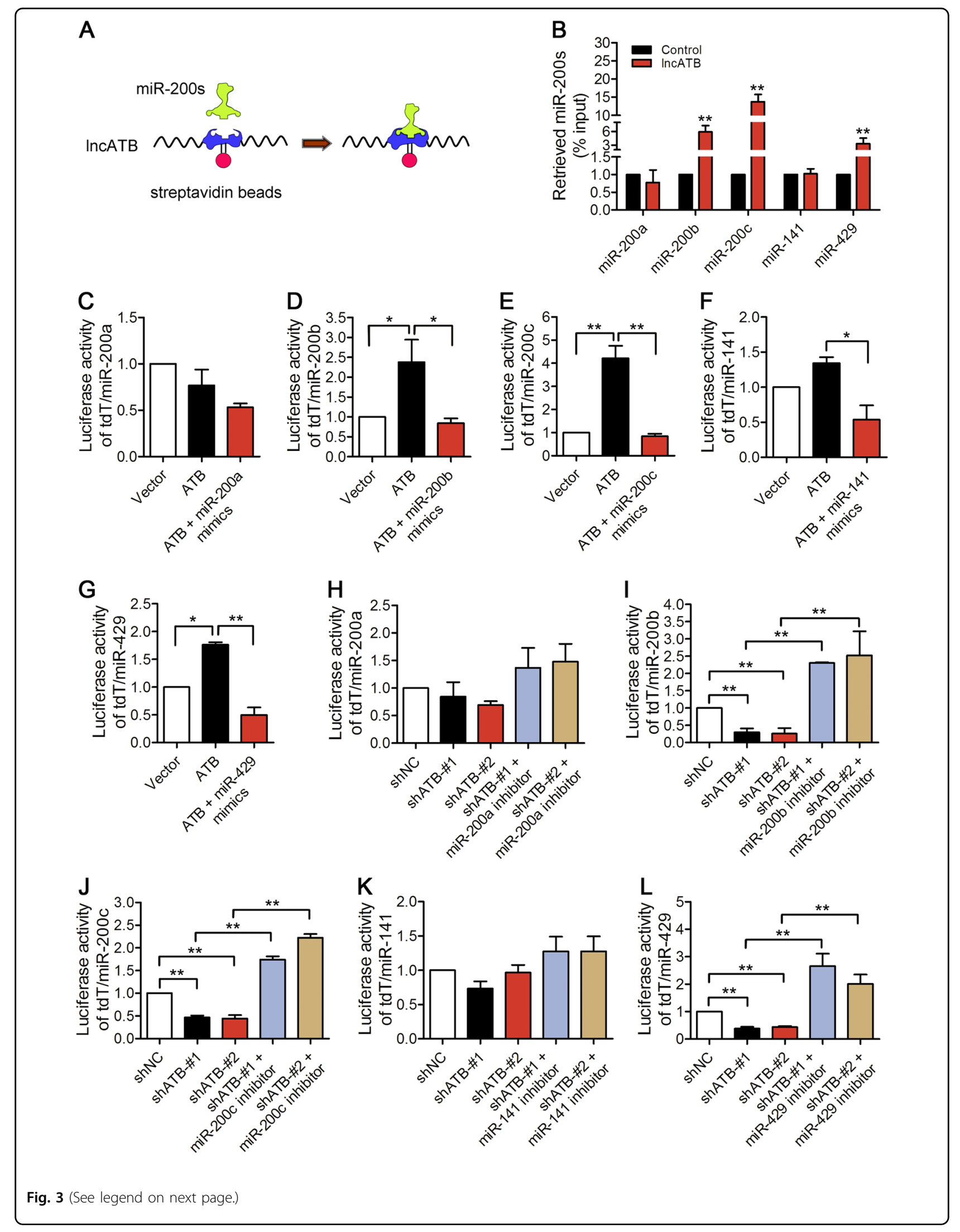


(see figure on previous page)

Fig. 3 LncATB functions by binding to miR-200 family members. a RNA pull-down followed by microRNA qRT-PCR assays to detect miR-200 family members endogenously associated with InCATB. b MCF-7 cell lysates were incubated with biotin-labelled IncATB; after the pull-down, miR-200 miRNAs were extracted and assessed by qRT-PCR assays. $\mathbf{c}-\mathbf{g}$ Relative luciferase activity in MCF-7 cells transfected with specific miR-200 luciferase reporters in the presence of pcDNA3.1-ATB alone or with the cotransfection of $\mathbf{c}$ miR-200a mimics, $\mathbf{d}$ miR-200b mimics, e miR-200c mimics, $\mathbf{f}$ miR-141 mimics, or $\mathbf{g}$ miR-429 mimics. $\mathbf{h}-\mathbf{I}$ Relative luciferase activity in BT-549 cells cotransfected with specific miR-200 luciferase reporters and shATB-\#1/2. For each specific reporter, $\mathbf{h}$ miR-200a inhibitor, i miR-200b inhibitor, $\mathbf{j}$ miR-200c inhibitor, $\mathbf{k}$ miR-141 inhibitor, or I miR-429 inhibitor was also added. ${ }^{*} P<0.05$ and ${ }^{*} P<0.01$

tumour weights than those in the control, with a $62 \%$ reduction observed in the shATB-\#1 group and a $46 \%$ reduction observed in the shATB-\#2 group (Fig. 6g). The immunohistochemistry results revealed that E-Cad expression was increased and Vimentin expression was decreased (Supplementary Fig. S3c). We extracted total RNA from the mouse tumour tissues, and the qRT-PCR assay results suggested that the ZEB1 and Twist1 expression levels were reduced (Supplementary Fig. S3d, e). These results indicated that lncATB downregulation inhibited tumour growth in vivo.

\section{LncATB is upregulated in breast cancer tissues and is associated with a poor prognosis in breast cancer patients}

Firstly, we searched the lncRNA database NONCODE and found that normal lncATB expression was higher in breast and ovarian tissues than in other organs (Supplementary Fig. S4a, b). To further define the role of lncATB in human breast cancer patients and the correlation between IncATB and Twist1, we measured the relative expression levels of $\operatorname{lncATB}$ and Twist1 in 146 samples, including 15 normal breast tissues and 131 breast cancer tissues. Clinical data on the lncATB (Fig. 7a, b) and Twist1 (Fig. 7d, e) expression level revealed that higher expression level observed in triplenegative and Luminal B (Her-2 positive) breast cancer, relative lower level in Luminal A breast cancer, and the lowest level in normal mammary glands. The diagnostic efficacy of lncATB (AUC $=0.851, P<0.001$, Fig. 7c) and Twist1 (AUC $=0.778, P=0.001$, Fig. $7 \mathrm{f}$ ) in breast cancer patients was evaluated by calculating the area under the receiver operating characteristic (ROC) curve. Moreover, we also found that the lncATB transcript level was significantly correlated with the Twist1 (Fig. 7g) mRNA levels. Patients were classified based on the level of lncATB expression in tissues into the low expression group $(n=39)$ and the high expression group $(n=92)$. Then, we analysed the disease-free survival (DFS) and the overall survival (OS) using a Kaplan-Meier analysis between the two groups. The results showed that the DFS $\left(\chi^{2}=4.099, P=0.043\right)$ and OS $\left(\chi^{2}=3.988, P=\right.$ 0.046) were longer in the low-lncATB-expression group than in the high-lncATB-expression group (Fig. 7h, i). However, there was no significant difference in the DFS or OS between the groups with high and low expression levels of Twist1 (Supplementary Fig. S5a, b). Similarly, we further evaluated the prognostic value of low or high expression of Twist1 mRNA in Kaplan-Meier Plotter database of breast cancer. The expression of Twist1 was not significantly related to RFS ( $P=0.7$, Supplementary Fig. S5c) and OS ( $P=0.31$, Supplementary Fig. S5d). In our study, we have further analysed the survival of both lncATB and Twist 1 in breast cancer patients. There was no statistically significant difference between DFS $(P=$ 0.174 , Supplementary Fig. S5e) and OS $(P=0.170$, Supplementary Fig. S5f), but the results tend to that breast cancer patients have a shorter survival when both lncATB and Twist1 were both positively expressed. Thus, these results indicate that lncATB might be a new poor prognostic marker for breast cancer with potential oncogenic characteristics.

\section{The clinicopathological features associated with low and high IncATB and Twist 1 expression in breast cancer patients \\ We further assessed the correlation between the} lncATB expression level and the clinicopathological characteristics of 131 breast cancer samples, as summarized in Table 1. The percentage of patients with histological grade III breast cancer was $67.4 \%$ in the highlncATB-expression group compared with $43.6 \%$ in the low-lncATB-expression group $\left(\chi^{2}=6.482 ; P=0.011\right)$. Additionally, the rate of Ki67 positivity was $92.4 \%$ in the high-IncATB-expression group and $56.4 \%$ in the lowlncATB-expression group $\left(\chi^{2}=23.696 ; P<0.001\right)$. In total, 18 out of 92 patients (19.6\%) with high lncATB expression exhibited a significantly higher rate of distant metastasis than 2 out of 39 patients (5.1\%) in the lowlncATB-expression group $\left(\chi^{2}=4.413, \quad \mathrm{P}=0.036\right)$. Although there were no significant differences in the clinical stage $(P=0.060)$ or the number of lymph node (LN) metastases $(P=0.051)$ between the low- and highlncATB-expression groups, we believe that there would be different results if we expanded the data. However, no significant differences in the years of age, tumour size or lymph node status were identified between the lowlncATB-expression group and the high-lncATBexpression group. 

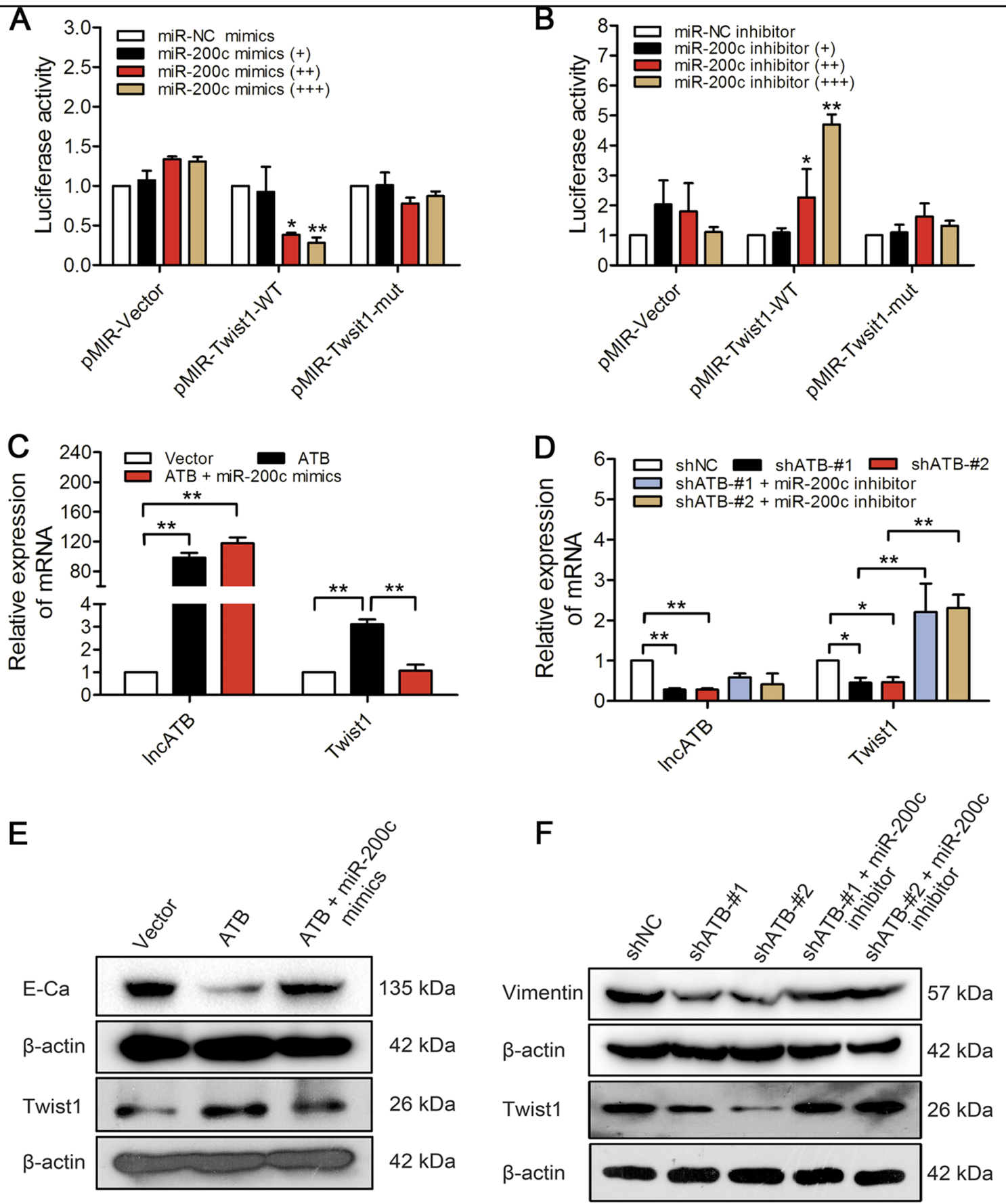

Fig. 4 Regulation of Twist1 mediated by IncATB via binding to miR-200c. a Luciferase activity in MCF-7 cells cotransfected with luciferase reporter (pMIR-Vector, pMIR-Twist1-WT, or pMIR-Twist1-mut) and miR-200c mimics with a dose dependent. b Luciferase activity in BT-549 cells cotransfected with luciferase reporter (pMIR-Vector, pMIR-Twist1-WT, or pMIR-Twist1-mut) and miR-200c inhibitor with a dose dependent. c The mRNA levels of Twist1 in MCF-7 cells overexpressing IncATB or ectopically expressing both IncATB and miR-200c. $\mathbf{d}$ The mRNA levels of Twist1 in BT549 cells with downregulated IncATB or with both shATB and miR-200c inhibitor. e The protein levels of Twist1 and E-Cad in MCF-7 cells overexpressing IncATB or ectopically expressing both IncATB and miR-200c. $\mathbf{f}$ The protein levels of Twist1 and Vimentin in BT-549 cells with downregulated InCATB or with both shATB and miR-200c inhibitor. ${ }^{*} P<0.05$ and ${ }^{* *} P<0.01$

Since we found that the sponging of miR-200c by lncATB restored the expression of Twist1 in breast cancer cell lines, we also investigated the correlation between the clinicopathological characteristics and Twist1 expression levels in breast cancer patients. As shown in Supplementary Tables S2, more breast cancer patients were 

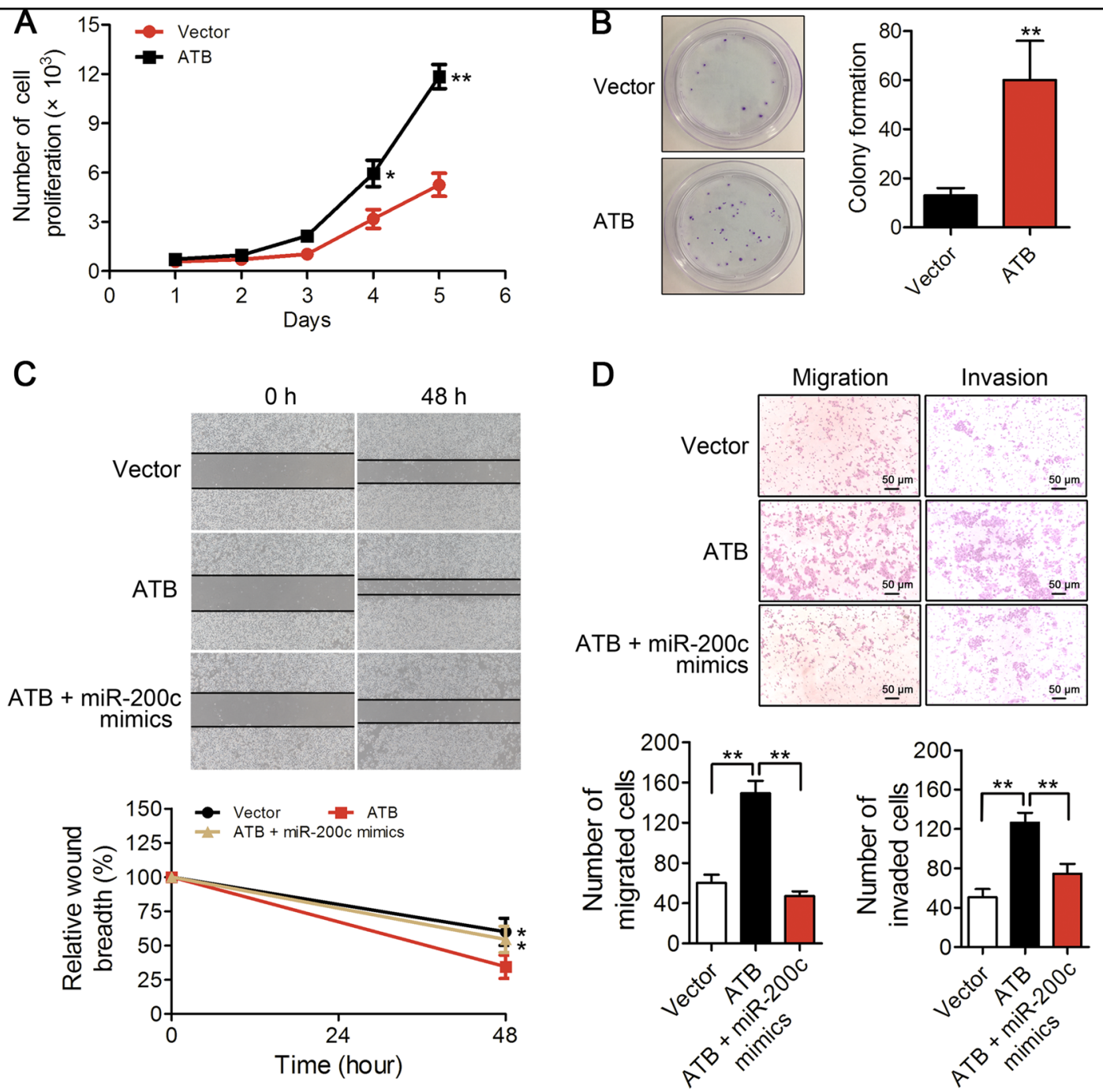

Fig. 5 LncATB promotes cancer progression in MCF-7 cells. a Cell proliferation of stably IncATB-overexpressing and control MCF-7 cells as assessed by the CCK-8 assay. $\mathbf{b}$ Colony numbers were counted in the MCF-7-ATB and control MCF-7 cell groups. $\mathbf{c}$ Wound-healing assay to evaluate the effect of IncATB on cell migration in groups of control MCF-7, MCF-7-ATB, or cells overexpressing both IncATB and miR-200c. $\mathbf{d}$ The migrated (left) and invaded (right) cell numbers for the groups of control MCF-7, MCF-7-ATB cells, or cells overexpressing both IncATB and miR-200c were counted $48 \mathrm{~h}$ after seeding. Scale bar $=50 \mu \mathrm{m} .{ }^{*}<0.05$ and ${ }^{* *}<0.01$

stage III or IV (39 out of $86,45.3 \%)$ when Twist1 displayed a high expression level, while fewer breast cancer patients were at these stages ( 7 out of $45,15.6 \%)$ when Twist1 displayed a low expression level $\left(\chi^{2}=10.239, P=\right.$ 0.001). Moreover, breast cancer patients were more likely to have lymph node metastasis when Twist1 was highly expressed $\left(\chi^{2}=7.523, P=0.023\right)$. Nevertheless, no significant differences in age, histology grade, tumour size, distant metastasis or Ki67 were identified between the low- and high-Twist1-expression groups.

\section{Discussion}

In the present study, we investigated the role of lncATB, an oncogenic lncRNA, in breast cancer cells and breast cancer patients, especially, its critical regulatory mechanism in breast cancer cell migration and cancer metastasis. We found that lncATB was induced by the TGF- $\beta$ treatment of breast cancer cells, whose induction is associated with EMT transition. Specifically, our study showed that lncATB could directly bind to the miR-200 family, with an especially high binding affinity for miR-200c. Moreover, lncATB regulated the gene expression of transcription factor Twist1, by acting as a competitive sponge for miR-200c and further promoted breast cancer cell migration and invasion (Fig. 8). Additionally, lncATB also increased breast cancer cell proliferation and promoted breast cancer cell clonality. 

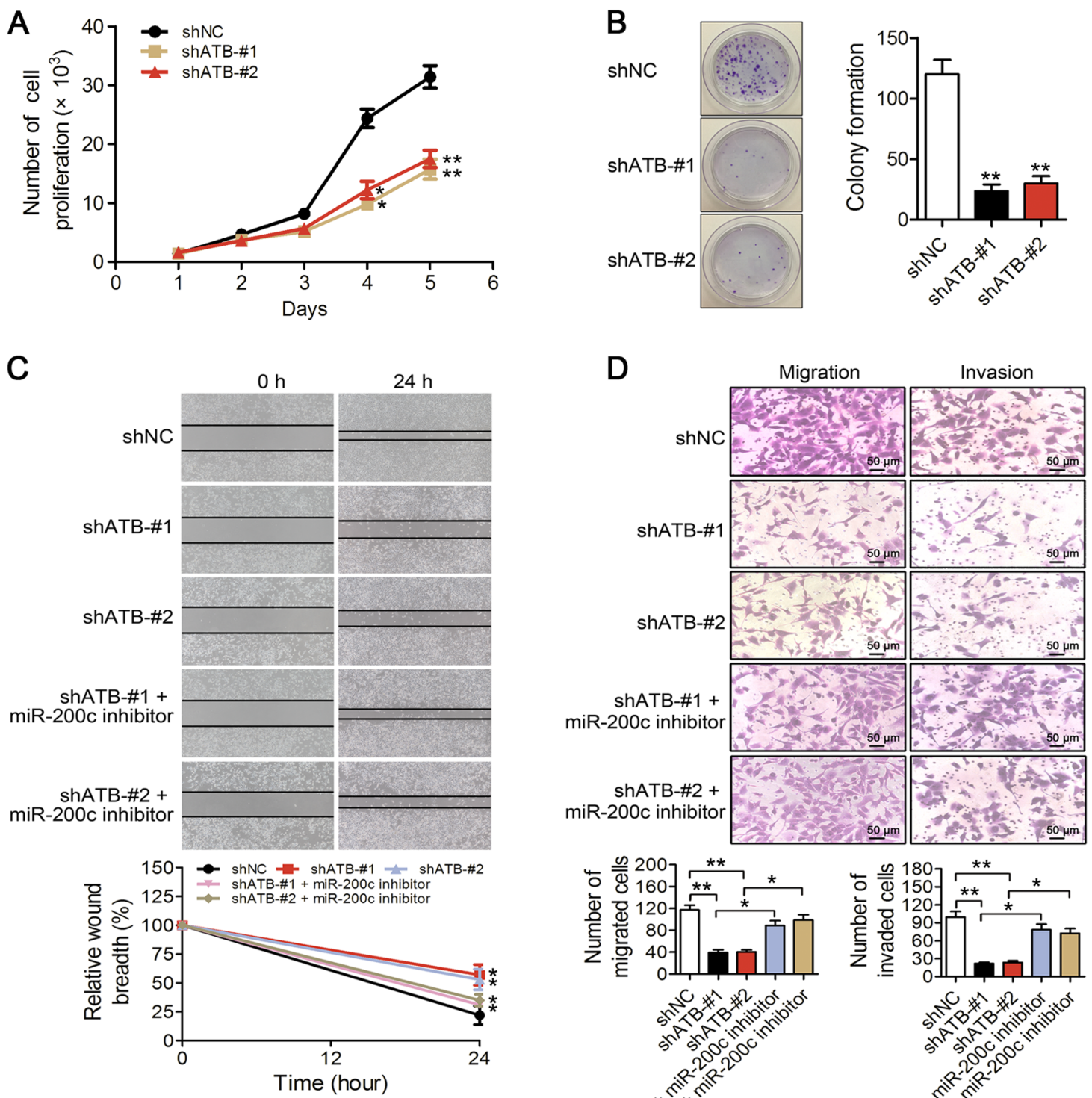

E

$\mathrm{F}$

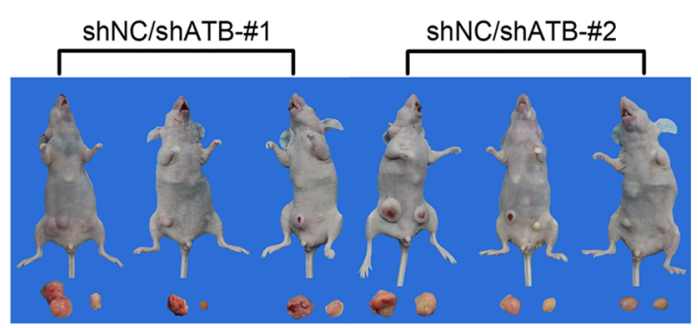

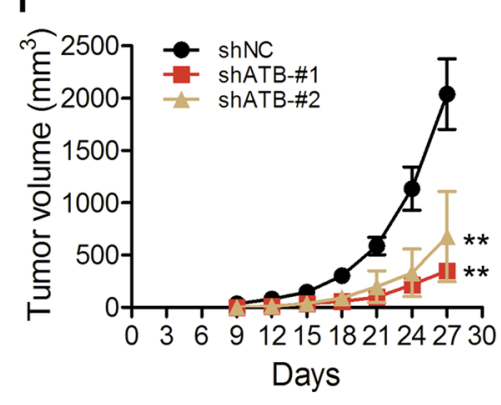

G

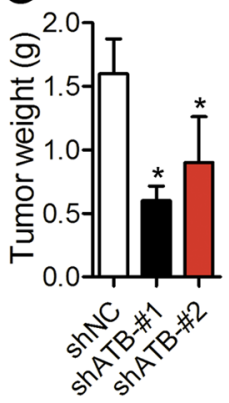

Fig. 6 (See legend on next page.) 
(see figure on previous page)

Fig. 6 LncATB deletion reduced breast cancer progression in vitro and in vivo. a Cell viability assay in BT-549 cells with knocked down IncATB. $\mathbf{b}$ Colony numbers were counted in the BT-549-shATB and control BT-549-shNC cell groups. c Wound-healing assay to evaluate the effect of IncATB on cell migration in the BT-549-shATB and control BT-549-shNC cell groups. $\mathbf{d}$ The migrated (left) and invaded (right) cell numbers in the groups of control BT-549-shNC cells, BT-549-shATB cells, and cells with both IncATB and miR-200c inhibition were counted $24 \mathrm{~h}$ after seeding. e For each mouse, shNC cells and IncATB-depleted cells were subcutaneously implanted into the left and right fourth mammary pad, respectively. There were two groups: shNC/shATB-\#1 and shNC/shATB-\#2, each group has three mice. $\mathbf{f}$ Tumours were measured every 3 days with external callipers, and the tumour volume was calculated according to the following formula: Volume $=0.5 \times a^{2} \times b$, where ' $a$ ' is the smallest superficial diameter and ' $b$ ' is the largest superficial diameter. $\mathbf{g}$ Twenty-seven days after the tumour was implanted, the tumours were excised and weighed. Scale bar $=50 \mu \mathrm{m}$. ${ }^{*} P<$ 0.05 and ${ }^{* *} P<0.01$

To date, several lncRNAs have been found to regulate breast cancer initiation and progression, and some of these lncRNAs including lncATB are potential prognostic markers for breast cancer ${ }^{25}$. Recent studies have indicated that lncATB is an essential modulator of cancer cell proliferation $^{36}$, the $\mathrm{EMT}^{32,36,43}$, anti-apoptosis ${ }^{33}$, migration and invasion ${ }^{32,34-36,42,43}$. A previous report demonstrated that lncATB promoted EMT-mediated trastuzumab resistance ${ }^{45}$. Consistent with that report, we also confirmed that lncATB promoted the EMT by inducing the expression of mesenchymal hallmarks, such as $\mathrm{N}$-Cad and Vimentin, and inhibiting the expression of the epithelial hallmark E-Cad in breast cancer cell lines. The ectopic expression of lncATB in breast cancer cells led to increased cellular proliferation, colony formation, migration and invasion, while lncATB knockdown inhibited those functions.

Emerging evidence has indicated that IncATB can promote cancer cell invasion and metastasis in a variety of cancer cells by acting as a competitive endogenous RNA (ceRNA) that acts as a sponge and modulates the functions of the miR-200 family by enhancing the expression of miR-200 target genes, such as ZEB1, ZEB2 or TGF$\beta 2^{32,34,42,43}$. In our study, we showed that, by sponging miR-200c, lncATB facilitated the expression of Twist1, resulting in promoted tumour progression. With regards to the miR-200 family members, miR-200c and then miR$200 \mathrm{~b}$ was highly inhibited with overexpression of lncATB, but rather than miR-200a as described in a previous report in $\mathrm{HCC}^{32}$. Several studies reveal that the member of miR-200 family showing the strongest binding ability with lncATB was different depending on tumour types such as miR-200a in liver cancer ${ }^{32}$, miR-200b in oesophageal squamous cell carcinoma ${ }^{41}$ and miR-141 in gastric cancer ${ }^{39}$. Together with our finding on the binding between miR-200c and lncATB in breast cancer, these findings suggest that lncATB might modulate different spectrum of miR-200s family. Probably in breast cancer, lncATB does mainly bind to miR-200c for silencing its effects on target genes.

Intriguingly, Twist 1 was another most significantly activated transcriptional factors by lncATB overexpression among those evaluated in breast cancers.
Twist1 was induced at both mRNA and protein levels by IncATB, while both of them were downregulated by depletion of lncATB. In contrast to the previous study that both ZEB1 and ZEB2 were activated in HCC, we found that Twist1 is another transcription factor induced by lncATB not reported previously. The positive association between IncATB and Twist1 was further confirmed in resected tissues from tumour xenografts. Notably, we also found that Twist1 expression correlated with lncATB in the tissues from breast cancer patients, keeping consistent with their correlation found in breast cancer cells. Taken together, these results suggested that lncATB promotes the EMT process by upregulating Twsit1. Remarkably, the results of mouse mammary-pad injection experiments with stable shATB-expressing MDA-MB-231 cells indicated the abolishment of breast cancer growth in vivo. All of these studies implied that lncATB might play a crucial role in breast cancer tumourigenesis and progression.

Several previous reports have shown that $\operatorname{lncATB}$ is upregulated in patients with cancer, especially metastatic cancer, including renal cell carcinoma ${ }^{36,37}$, hepatocellular carcinoma ${ }^{32}$, non-small cell lung cancer $^{33}$, osteosarcoma ${ }^{34}$, colon cancer ${ }^{35}$, gastric cancer ${ }^{38,39}$, oesophageal squamous cell carcinoma ${ }^{41}$, glioma malignancy ${ }^{42}$ and prostate carcinoma ${ }^{43}$. In the current study, lncATB and its downstream gene Twist1 have been applied to the association with the clinicopathological characteristics and survival of breast cancer patients. Notably, for the first time, we demonstrated that lncATB was highly expressed in clinical breast cancer tissues, especially in luminal $\mathrm{B}, \mathrm{Her}-2$ and TNBC subtypes compared to the luminal A subtype, which keeps consistent with that found in breast cancer cells. Furthermore, high levels of lncATB in breast cancer patients were associated with more distant metastasis, higher histological grade, more lymph nodes involvement, and higher Ki67 levels, i.e., high aggressiveness of breast cancers. In addition, the patients with high lncATB level showed a poor clinical outcome including both DFS and OS, similar to those found in carcinomas ${ }^{34,37,38,42,43,46,47}$. Taken together, the findings here demonstrated that lncATB associates with unfavourable biological behaviour and predicts poor 

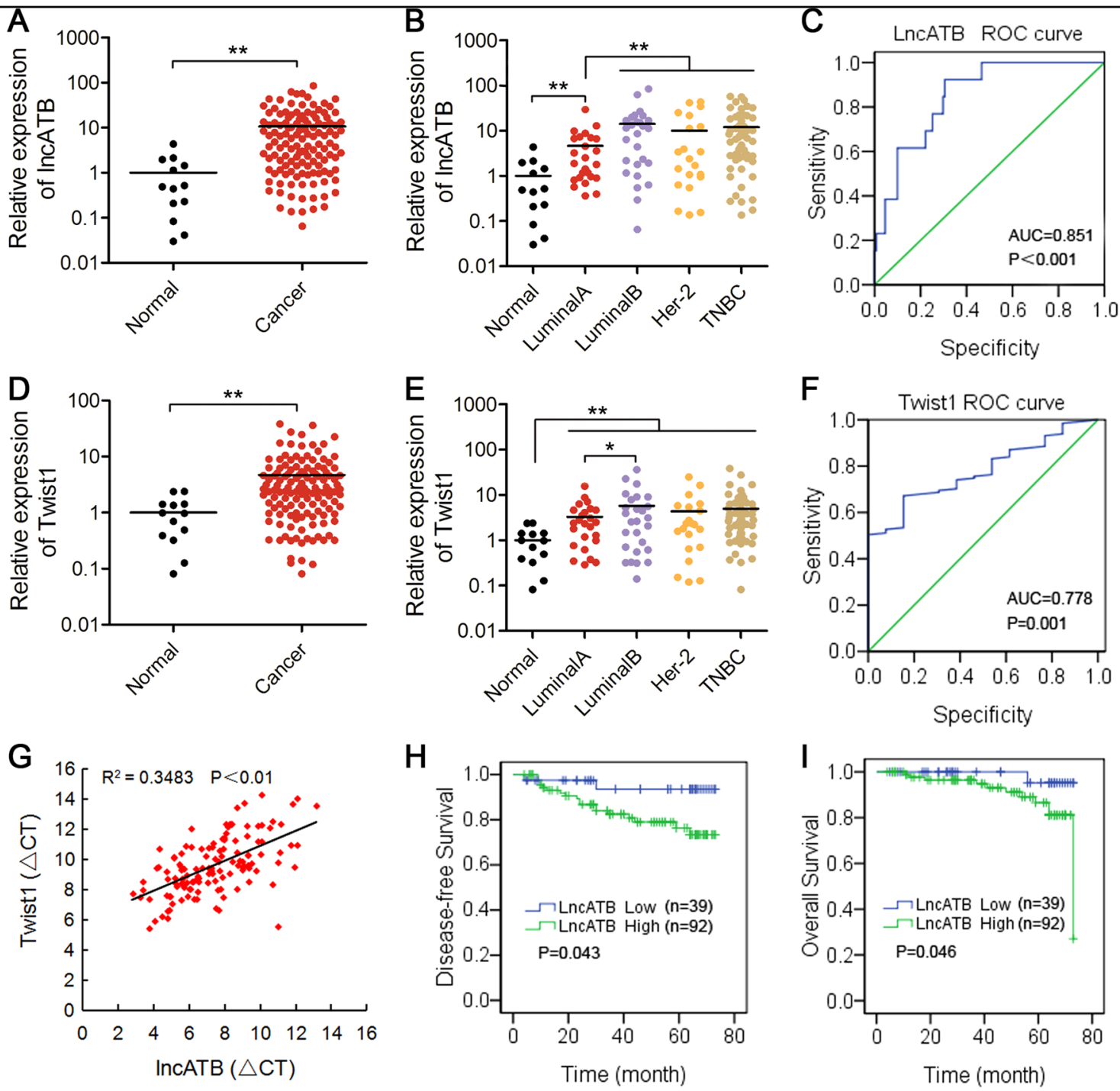

Fig. 7 High levels of IncATB indicated poor prognosis in breast cancer patients. a The relative expression of IncATB in normal breast and breast cancer tissues. $\mathbf{b}$ The relative expression of IncATB in different breast cancer tissue subtypes. c Assessment of the diagnostic efficacy of IncATB in breast cancer patients by calculating the area under the ROC curve (AUC $=0.851, P<0.001$ ). $\mathbf{d}$ The relative expression of Twist 1 in normal breast and breast cancer tissues. $\mathbf{e}$ The relative expression of Twist1 in different breast cancer tissue subtypes. $\mathbf{f}$ Assessment of the diagnostic efficacy of Twist1 in breast cancer patients by calculating the area under the ROC curve (AUC $=0.778, P=0.001$ ). $\mathbf{g}$ The correlation between the IncATB transcript level and Twist1 mRNA levels was measured in 131 breast cancer tissues. $\mathbf{h}$ Disease-free survival for the two groups defined by low and high expression of IncATB in breast cancer patients. $\mathbf{i}$ Overall survival for the two groups defined by low and high expression of IncATB in breast cancer patients. ${ }^{*} P<$ 0.05 and ${ }^{* *} P<0.01$

prognosis in breast cancers. Additionally, our study also showed that the AUC of the ROC analysis of lncATB was as high as 0.851 in breast cancer patients, indicating the diagnostic value of lncATB for cancer. Collectively, the ability of lncATB to predict poor clinical outcomes is promising, and the results suggest that lncATB may be used as a novel marker in cancer diagnosis and predicting prognosis.

In conclusion, we have described the important regulatory roles played by the $\operatorname{lncATB} / \mathrm{miR} 200 \mathrm{c} / \mathrm{Twist} 1$ axe in breast cancer carcinogenesis and progression. Our study suggests that addressing the uncharted potential effects of lncATB will have a fundamental influence on breast cancer biology, especially in the search for novel diagnostic markers for breast cancer as well as new therapeutic targets and pharmaceutical interventions.

\section{Materials and methods \\ Patients}

A total of 131 primary breast cancer tissue samples and 16 normal breast tissue samples were collected from patients who underwent surgeries in the Breast Center at 
Table 1 Clinicopathological characteristics of the breast cancer patients according to IncATB expression

\begin{tabular}{|c|c|c|c|c|}
\hline \multirow[t]{2}{*}{ Characteristics } & \multicolumn{2}{|c|}{ IncATB expression } & \multirow[t]{2}{*}{$x^{2}$} & \multirow[t]{2}{*}{$P$ value } \\
\hline & High $n(\%)$ & Low $n(\%)$ & & \\
\hline \multicolumn{5}{|l|}{ Age years } \\
\hline$\leq 40$ & $14(15.2)$ & $4(10.3)$ & 0.569 & 0.451 \\
\hline$>40$ & $78(84.8)$ & 35 (89.7) & & \\
\hline \multicolumn{5}{|l|}{ Histology grade } \\
\hline | and || & $30(32.6)$ & $22(56.4)$ & 6.482 & 0.011 \\
\hline III & $62(67.4)$ & $17(43.6)$ & & \\
\hline \multicolumn{5}{|l|}{ Stage } \\
\hline | and || & $55(59.8)$ & $30(76.9)$ & 3.532 & 0.060 \\
\hline III and IV & $37(40.2)$ & $9(23.1)$ & & \\
\hline \multicolumn{5}{|l|}{ Tumour size } \\
\hline T0-T1 & $20(21.7)$ & $9(23.1)$ & 0.028 & 0.866 \\
\hline $\mathrm{T} 2-\mathrm{T} 4$ & $72(78.3)$ & $30(76.9)$ & & \\
\hline \multicolumn{5}{|l|}{ LN status } \\
\hline Negative & $38(41.3)$ & 19 (48.7) & 0.612 & 0.434 \\
\hline Positive & $54(58.7)$ & $20(51.3)$ & & \\
\hline \multicolumn{5}{|c|}{ No. of LN metastasis } \\
\hline$L N \leq 3$ & $63(68.5)$ & $31(79.5)$ & 5.965 & 0.051 \\
\hline $3<\mathrm{LN}<10$ & $12(13.0)$ & $7(17.9)$ & & \\
\hline$L N \geq 10$ & $17(18.5)$ & $1(2.6)$ & & \\
\hline \multicolumn{5}{|l|}{ Distant metastasis } \\
\hline Negative & $74(80.4)$ & $37(94.9)$ & 4.413 & 0.036 \\
\hline Positive & 18 (19.6) & $2(5.1)$ & & \\
\hline \multicolumn{5}{|l|}{ Ki67 } \\
\hline Negative & $7(7.6)$ & 17 (43.6) & 23.696 & $<0.001$ \\
\hline Positive & $85(92.4)$ & $22(56.4)$ & & \\
\hline
\end{tabular}

These bold values indicate there is statistical significance between the two groups, $P<0.05$ or $P<0.01$.

the Cancer Hospital of Shantou University Medical College between September 2010 and June 2016. Tissue specimens obtained during surgery were immediately snap-frozen in liquid nitrogen, and RNA was extracted within $48 \mathrm{~h}$. None of the patients had received preoperative chemotherapy, radiotherapy, endocrine therapy, or targeted therapy. Clinicopathological parameters, such as the tumour size, lymph node status, and pathological features, were recorded from the patients' medical records. The study was approved by the ethics committee of the Cancer Hospital of Shantou University Medical College and written informed consent was obtained from all patients involved.

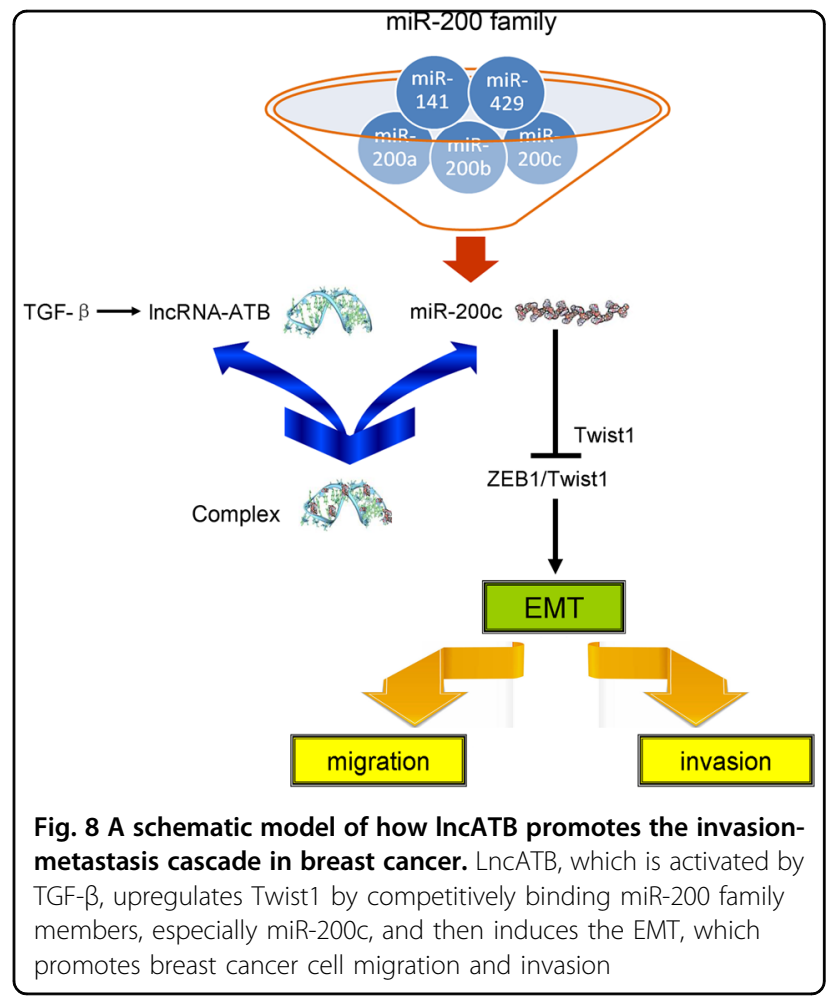

\section{Cell lines and cell culture}

The MCF-7, T47D, MDA-MB-231, BT-549, BT-20 MDA-MB-436, MDA-MB-435, and SKBR3 breast cancer cell lines and MCF-10A normal breast cells were purchased from the American Type Culture Collection (ATCC, USA), which then were authenticated by STR (Short Tandem Repeat) profiling and tested for mycoplasma-free contamination. All the cells were maintained in dulbecco's modified eagle medium (DMEM) containing 10\% foetal bovine serum (FBS) (Gibco/Life Technologies, Carlsbad, CA, USA) and were cultured in a humidified $5 \% \mathrm{CO}_{2}$ incubator at $37^{\circ} \mathrm{C}$. The medium was replaced every 2 days.

\section{RNA extraction and real-time qPCR}

Total RNA from all the tissues and cells was extracted with the TRIzol reagent (Life Technologies, Gaithersburg, MD, USA). The RNA was reverse-transcribed into cDNA using the Prime-Script RT-PCR kit (Takara, Dalian, China) following the manufacturer's instructions. All the primer sequences are shown in Supplementary Table S3. The transcript levels of lncATB and the EMT markers were analysed using real-time PCR assays (SYBR Premix Ex Taq ${ }^{\mathrm{Tm}}$, TaKaRa, Dalian, China) in triplicate by reacting the cDNA with sequence-specific PCR primers in an ABI PRISM 7500 sequence detection PCR system (Applied Biosystems, Foster City, CA, USA). The relative expression levels of lncATB and the mRNAs were calculated and normalized relative to $\beta$-actin using the $2-\Delta \Delta \mathrm{Ct}$ method. 


\section{RNA pull-down assay}

RNA pull-down assays were performed according to the manufacturer's instructions (Sagene Technology Inc., Guangzhou, China). We labelled lncATB probes with biotin using transcription and then incubated the probes with an MCF-7 cytoplasmic lysate to form lncRNAmicroRNA complexes. The complexes were combined via chain affinity with magnetic beads and thus separated from other components. After complex elution, we determined by qRT-PCR assays the miR-200 family members that were pulled down.

\section{Western blot analysis}

The total cellular proteins were lysed in RIPA buffer (Cell Signaling Technology, Inc., MA, USA) supplemented with protease inhibitors. The protein concentration was determined using a BCA Protein Assay Kit (Beyotime Biotechnology, Shanghai, China). Extracted protein $(30-50 \mu \mathrm{g})$ was separated by $10 \%$ SDS polyacrylamide gel electrophoresis and transferred to a polyvinylidene difluoride membrane (EMD Millipore Corporation, Billerica, MA, USA). The membrane was blocked with $5 \%$ non-fat milk in Tris-buffered saline with $0.1 \%$ Tween-20 (TBST) for $1 \mathrm{~h}$ and then probed with the indicated primary antibodies (Supplementary Table S4) at $4{ }^{\circ} \mathrm{C}$ overnight with gentle shaking. The following day, the membrane was washed with TBST $(5 \min \times 3)$ and then incubated in secondary antibody (Supplementary Table S5) for $2 \mathrm{~h}$ at room temperature. Then the immunocomplexes were detected using the ECL Plus reagent (Applygen Technologies, Inc., Beijing, China).

\section{Immunofluorescence assay}

MCF-7 or BT-549 cells were plated on glass slides $(1 \times$ $10^{4}$ cells/well) and incubated for approximately $24 \mathrm{~h}$ at $37^{\circ} \mathrm{C}$. After treating the cells with formaldehyde and Triton- $\mathrm{X}$, the cells were incubated with primary antibodies directed against E-Cad and Vimentin (dilution 1:250, Cell Signaling Technology, Inc., USA) at $4{ }^{\circ} \mathrm{C}$ overnight with gentle shaking. The following day, the cells were incubated with secondary antibody (Supplementary Table S5) for $1 \mathrm{~h}$ at room temperature away from light sources. Finally, the samples were stained with 4',6-diamidino-2-phenylindole (DAPI) and were observed with a TS100/100-F inverted fluorescence microscope (Nikon, Corporation, Tokyo, Japan).

\section{Cell proliferation assay}

Cells were seeded into 96-well plates at a density of $1 \times 10^{3}$ cells/well and incubated for $1-5$ days. Every $24 \mathrm{~h}$, $10 \mu \mathrm{l}$ of Cell Counting Kit-8 solution (CCK-8, Dojindo, Japan) was added to each well, and the plates were incubated at $37^{\circ} \mathrm{C}$ in a humidified atmosphere containing
$5 \% \mathrm{CO}_{2}$ for $2 \mathrm{~h}$. Then, the optical density (OD) of each sample was determined by measuring the absorbance at $490 \mathrm{~nm}$ with a microplate reader (BioTek Instruments, Inc., Winooski, VT, USA).

\section{Colony formation assay}

Approximately $1 \times 10^{3}$ viable cells were seeded into 100 -mm plates and incubated in DMEM with $10 \%$ FBS at $37^{\circ} \mathrm{C}$. After 14 days, when the colonies were larger than 50 cells, the cells were stained using crystal violet. Each experiment was repeated twice.

\section{Wound-healing assay}

Cells $\left(5 \times 10^{5}\right)$ were seeded into six-well plates and allowed to grow to $90-95 \%$ confluence. Subsequently, the cells were scraped with a $200-\mu$ l plastic tip to generate straight wounds. At 0 and $48 \mathrm{~h}$ after wounding, images were recorded with an inverted research eclipse TS100/ 100-F microscope (Nikon, Corp., Tokyo, Japan).

\section{Cell migration and invasion assay}

Cell migration was determined by using 24-well Transwell Chambers with an $8-\mu \mathrm{m}$ pore size and a track-etched membrane (Corning, Inc., New York, CA, USA). A chamber insert was placed into each well of a 24well dish containing $600 \mu \mathrm{l}$ of DMEM supplemented with $20 \%$ FBS in the lower chamber. Approximately $5 \times 10^{4}$ cells were suspended in $200 \mu \mathrm{l}$ of serum-free DMEM and seeded into the upper chamber. The cells were incubated at $37{ }^{\circ} \mathrm{C}$ with $5 \% \mathrm{CO}_{2}$ for $24 \mathrm{~h}$. Then, the cells on the lower chamber membrane were fixed with $100 \%$ methanol for $30 \mathrm{~min}$ at room temperature and stained with $0.1 \%$ crystal violet for $20 \mathrm{~min}$ at room temperature. Subsequently, the nonmigrated cells on the upper side of the membranes were removed, and the migrated cells on the underside of the membranes were observed with a TS100/100-F inverted fluorescence microscope (Nikon, Corporation, Tokyo, Japan) using five randomized fields. A cell invasion assay was also conducted in the same manner but with Matrigel $^{\text {mit }}$ Invasion Chamber 24-well plates with $8.0-\mu \mathrm{m}$ pores (BD Biosciences, San Jose, USA) and an incubation time of $48 \mathrm{~h}$.

\section{RNA interference}

The miR-200 family member mimics and inhibitor were synthesized by GenePharma (Shanghai GenePharma Co., Ltd., Shanghai, China). The siRNA sequences for the miR200 family members are listed in Supplementary Table S6. The day prior to transfection, $5 \times 10^{5}$ MCF- 7 or BT- 549 cells were inoculated into each well of a six-well culture plate. For the transfection, 75 pmol of siRNA was combined with $5 \mu \mathrm{l}$ of Lipofectamine 3000 (Invitrogen; Thermo Fisher Scientific, Inc., Waltham, MA, USA) according to the manufacturer's protocol. 


\section{Tumour xenografts}

All animal studies were approved by the Institutional Animal Care and Use Committee of Shantou University Medical College and were performed with 4-week-old female nude mice (Vital River Laboratory Animal Technology Co., Ltd., Beijing, China). A total of $2 \times 10^{6}$ cells were suspended in $100 \mu \mathrm{l}$ of phosphate buffer saline (PBS) and injected into the fourth breast of three female nude mice in each group. Tumour growth was determined, along the longest diameter and shortest width, every 3 days using digital callipers. The tumour volume was calculated according to the following formula: Volume $=$ $0.5 \times$ width $^{2} \times$ length. Finally, the tumours were removed, measured and weighed. For xenograft animal studies, the protocol was approved by the Animal Care and Use Committee of Shantou University Medical College.

\section{Vector and shRNA construction}

The cDNA encoding lncATB was amplified via PCR with the TransStart FastPfu DNA Polymerase (TransGen Biotech, Beijing, China, \#J30702). Then, the lncATB sequence was subcloned into the XhoI and EcoRI sites of the pcDNA3.1 (+) vector, which was named pcDNA3.1ATB (ATB) (Supplementary Fig. S1a). The lncATB primers were $5^{\prime}$-CCCTCGAGCCCTGGGGCTCTGCAA TTG-3' (forward) and $5^{\prime}$-CGGAATTCGGTAAAT GAGTCCAAAG TCATACTGCCC-3' (reverse).

The design of shATB was assisted by GenePharma (Shanghai GenePharma Co., Ltd., Shanghai, China) and included the following primers: shATB-\#1, 5'CCTTATGGCCTAGATTACCTTTCCAT-3'; shATB-\#2, 5'-CCTGTCTGTATTT GCGAATACCTT-3'; and shNC, $5^{\prime}$-T TCTCCGAACGTGTCACGT- $3^{\prime}$. The shATB sequences were cloned into pGPU6/GFP/Neo plasmids, which are driven by a U6 promoter.

\section{Luciferase reporter assays}

The IncATB fragment containing the predicted miR200 family member binding site was obtained, and the putative binding site sequences were cloned into a pcDNA3.1(+)/Luc2 $=$ tdT vector (Addgene, Sidney St., Cambridge, MA) (Supplementary Fig. S6a). The twist1-3' UTR fragment sequence containing the binding site with miR-200c was cloned into the pMIR-reporter vector (Addgene, Sidney St., Cambridge, MA) (Supplementary Fig. S6b). Cells were cotransfected with luciferase reporters and other plasmids or short RNAs (ATB, shATB, miR-200 family mimics, or the miR-200 family inhibitor) using Lipofectamine 3000 (Invitrogen; Thermo Fisher Scientific, Inc., Waltham, MA, USA). After $48 \mathrm{~h}$, a luciferase assay was performed using the Dual-Luciferase Reporter Assay System (Promega, Madison, WI, USA) according to the manufacturer's protocol.

\section{Statistical analysis}

Statistical analysis was performed using the SPSS version 20.0 (SPSS, Inc., Chicago, IL, USA) and Prism V5.0 (GraphPad Software, Inc., La Jolla, CA, USA) software. The experimental data were presented as the means \pm standard deviation (SD). Statistical analysis was performed using Student's $t$ test. The Spearman correlation coefficient was used to assess correlations between lncATB expression and clinical features. The survival analysis was performed using the log-rank test in GraphPad Prism 5. A value of $P<0.05$ was considered to indicate a statistically significant difference. The corresponding significance levels are indicated in the figures.

\section{Acknowledgements}

The present study was supported by the Key International Collaborative Project of National Natural Science Fund Committee (grant no. 81320108015), the National Natural Science Fund project (grant no. 31271068), the Special Research Fund for the Doctoral Program of Higher Education (grant no. 201244021 10003), and the Start-Up Fund for high-end talents from Xiamen University (G.J.Z.).

\section{Author details \\ ${ }^{1}$ Chang Jiang Scholar's Laboratory, Shantou University Medical College (SUMC) 515041 Shantou, Guangdong, China. ${ }^{2}$ The Cancer Center, Xiang'an Hospital of Xiamen University, 2000 East Xiang'an Rd., Xiang'an, Xiamen, China. ${ }^{3}$ The Breast Center, The Cancer Hospital of SUMC, 515041 Shantou, Guangdong, China. ${ }^{4}$ Department of Pathology, The Cancer Hospital of SUMC, Shantou, Guangdong, China}

Conflict of interest

The authors declare that they have no conflict of interest.

\section{Publisher's note}

Springer Nature remains neutral with regard to jurisdictional claims in published maps and institutional affiliations.

Supplementary Information accompanies this paper at (https://doi.org/ 10.1038/s41419-018-1210-9).

Received: 22 June 2018 Revised: 27 October 2018 Accepted: 5 November 2018

Published online: 05 December 2018

\footnotetext{
References

1. Matsumoto, A., et al. Biological markers of invasive breast cancer. Jpn. J. Clin. Oncol. 46, 99-105 (2016).

2. Ward, E. M. et al. Cancer statistics: breast cancer in situ. CA Cancer J. Clin. 65 481-495 (2015).

3. DeSantis, C. et al. Breast cancer statistics, 2013. CA Cancer J. Clin. 64, 52-62 (2014).

4. Castrellon, A. B. Novel strategies to improve the endocrine therapy of breast cancer. Oncol. Rev. 11, 323 (2017).

5. Hu, X., Huang, W. \& Fan, M. Emerging therapies for breast cancer. J. Hematol. Oncol. 10, 98 (2017)

6. Huarte, M. The emerging role of IncRNAs in cancer. Nat. Med. 21, 1253-1261 (2015).

7. Bartonicek, N., Maag, J. L. \& Dinger, M. E. Long noncoding RNAs in cancer: mechanisms of action and technological advancements. Mol. Cancer 15, 43 (2016).

8. Bolha, L., Ravnik-Glavac, M. \& Glavac, D. Long noncoding RNAs as biomarkers in cancer. Dis. Markers 2017, 7243968 (2017).
} 
9. Losko, M., Kotlinowski, J. \& Jura, J. Long noncoding RNAs in metabolic syndrome related disorders. Mediat. Inflamm. 2016, 5365209 (2016).

10. Soudyab, M., Iranpour, M. \& Ghafouri-Fard, S. The role of long non-coding RNAs in breast cancer. Arch. Iran. Med. 19, 508-517 (2016).

11. Mondal, T. et al. MEG3 long noncoding RNA regulates the TGF-beta pathway genes through formation of RNA-DNA triplex structures. Nat. Commun. 6 , 7743 (2015).

12. Rinn, J. L. et al. Functional demarcation of active and silent chromatin domains in human HOX loci by noncoding RNAs. Cell 129, 1311-1323 (2007).

13. Schmitz, S. U., Grote, P. \& Herrmann, B. G. Mechanisms of long noncoding RNA function in development and disease. Cell. Mol. Life Sci. 73, 2491-2509 (2016).

14. Schmitt, A. M. \& Chang, H. Y. Long noncoding RNAs in cancer pathways. Cancer Cell 29, 452-463 (2016).

15. Dhamija, S. \& Diederichs, S. From junk to master regulators of invasion: IncRNA functions in migration, EMT and metastasis. Int. J. Cancer 139, 269-280 (2016).

16. Sorensen, K. P. et al. Long non-coding RNA HOTAIR is an independent prognostic marker of metastasis in estrogen receptor-positive primary breast cancer. Breast Cancer Res. Treat. 142, 529-536 (2013).

17. Tao, S., He, H. \& Chen, Q. Estradiol induces HOTAIR levels via GPER-mediated miR-148a inhibition in breast cancer. J. Transl. Med. 13, 131 (2015).

18. Latorre, E. et al. The ribonucleic complex HuR-MALAT1 represses CD133 expression and suppresses epithelial-mesenchymal transition in breast cancer. Cancer Res. 76, 2626-2636 (2016).

19. Meseure, D. et al. Prognostic value of a newly identified MALAT1 alternatively spliced transcript in breast cancer. Br. J. Cancer 114, 1395-1404 (2016).

20. Zhou, W. et al. The IncRNA H19 mediates breast cancer cell plasticity during EMT and MET plasticity by differentially sponging miR-200b/c and let-7b. Sci. Signal. 10, 483 (2017).

21. Raveh, E. et al. The $\mathrm{H} 19$ long non-coding RNA in cancer initiation, progression and metastasis - a proposed unifying theory. Mol. Cancer 14, 184 (2015)

22. Vennin, $\mathrm{C}$. et al. H19 non coding RNA-derived miR-675 enhances tumorigenesis and metastasis of breast cancer cells by downregulating c-Cbl and Cbl-b. Oncotarget 6, 29209-29223 (2015).

23. Di Gesualdo, F., Capaccioli, S. \& Lulli, M. A pathophysiological view of the long non-coding RNA world. Oncotarget 5, 10976-10996 (2014).

24. Godinho, M. F. et al. Relevance of BCAR4 in tamoxifen resistance and tumour aggressiveness of human breast cancer. Br. J. Cancer 103, 1284-1291 (2010).

25. Xu, S. et al. Oncogenic long noncoding RNA landscape in breast cancer. Mol. Cancer 16, 129 (2017).

26. LV, M. et al. LnCRNAs as new biomarkers to differentiate triple negative breast cancer from non-triple negative breast cancer. Oncotarget 7, 13047-13059 (2016).

27. Godinho, M. F. et al. BCAR4 induces antioestrogen resistance but sensitises breast cancer to lapatinib. Br. J. Cancer 107, 947-955 (2012).

28. Xing, Z. et al. LncRNA BCAR4 wires up signaling transduction in breast cancer. Rna Biol. 12, 681-689 (2015).

29. Van Grembergen, O. et al. Portraying breast cancers with long noncoding RNAs. Sci. Adv. 2, e1600220 (2016).
30. Tordonato, C., Di Fiore, P. P. \& Nicassio, F. The role of non-coding RNAs in the regulation of stem cells and progenitors in the normal mammary gland and in breast tumors. Front. Genet. 6, 72 (2015).

31. Amorim, M. et al. Decoding the usefulness of non-coding RNAs as breast cancer markers. J. Transl. Med. 14, 265 (2016).

32. Yuan, J. H. et al. A long noncoding RNA activated by TGF-beta promotes the invasion-metastasis cascade in hepatocellular carcinoma. Cancer Cell 25, 666-681 (2014).

33. Dykes, I. M. and Emanueli, C. Transcriptional and post-transcriptional gene regulation by long non-coding RNpA. Genom. Proteom. Bioinforma. 15, 177-186 (2017)

34. Han, F. et al. Long noncoding RNA ATB promotes osteosarcoma cell proliferation, migration and invasion by suppressing miR-200s. Am. J. Cancer Res. 7, 770-783 (2017).

35. Yue, B. et al. LncRNA-ATB mediated E-cadherin repression promotes the progression of colon cancer and predicts poor prognosis. J. Gastroenterol. Hepatol. 31, 595-603 (2016).

36. Xiong, J. et al. High expression of long non-coding RNA IncRNA-ATB is correlated with metastases and promotes cell migration and invasion in renal cell carcinoma. Jpn. J. Clin. Oncol. 46, 378-384 (2016).

37. Qi, J. J., Liu, Y. X. \& Lin, L. High expression of long non-coding RNA ATB is associated with poor prognosis in patients with renal cell carcinoma. Eur. Rev. Med. Pharmacol. Sci. 21, 2835-2839 (2017).

38. Saito, T. et al. A long non-coding RNA activated by transforming growth factor-beta is an independent prognostic marker of gastric cancer. Ann. Surg. Oncol. 22(Suppl 3), S915-S922 (2015).

39. Lei, K. et al. Lnc-ATB contributes to gastric cancer growth through a MiR-141$3 \mathrm{p} /$ TGFbeta2 feedback loop. Biochem. Biophys. Res. Commun. 484, 514-521 (2017).

40. Fu, X. M. et al. The expression and function of long noncoding RNA IncRNAATB in papillary thyroid cancer. Eur. Rev. Med. Pharmacol. Sci. 21, 3239-3246 (2017).

41. Li, Z. et al. Long non-coding RNA ATB promotes malignancy of esophageal squamous cell carcinoma by regulating miR-200b/Kindlin-2 axis. Cell Death Dis. 8, e2888 (2017)

42. Ma, C. C. et al. Long non-coding RNA ATB promotes glioma malignancy by negatively regulating miR-200a. J. Exp. Clin. Cancer Res. 35, 90 (2016).

43. Xu, S. et al. Long non-coding RNA ATB promotes growth and epithelialmesenchymal transition and predicts poor prognosis in human prostate carcinoma. Oncol. Rep. 36, 10-22 (2016).

44. Jang, S. Y. et al. Clinical significance of IncRNA-ATB expression in human hepatocellular carcinoma. Oncotarget 8, 78588-78597 (2017).

45. Shi, S. J. et al. LncRNA-ATB promotes trastuzumab resistance and invasionmetastasis cascade in breast cancer. Oncotarget 6, 11652-11663 (2015).

46. Iguchi, $T$. et al. A long noncoding RNA, InCRNA-ATB, is involved in the progression and prognosis of colorectal cancer. Anticancer Res. 35, 1385-1388 (2015).

47. Qu, S. et al. Downregulation of IncRNA-ATB correlates with clinical progression and unfavorable prognosis in pancreatic cancer. Tumour Biol. 37, 3933-3938 (2016). 